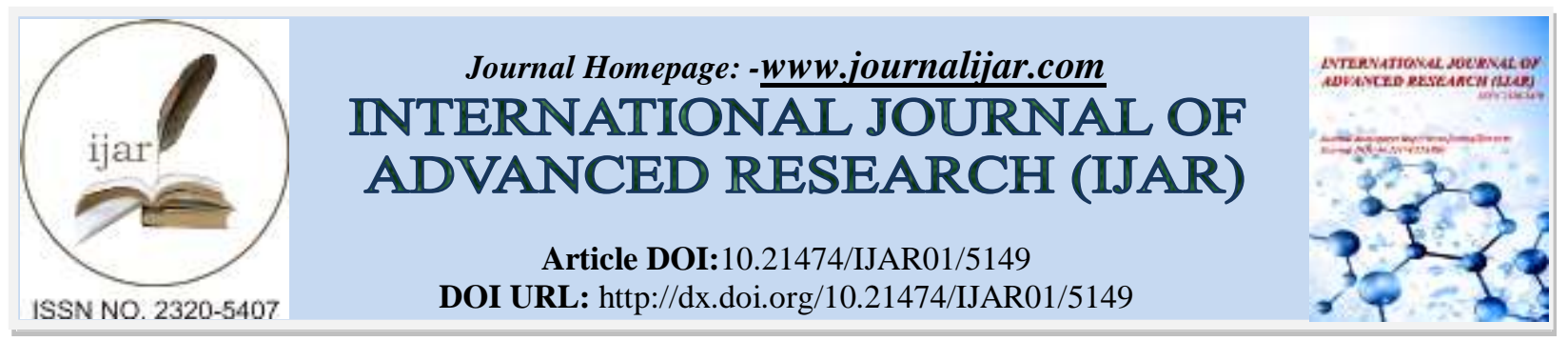

RESEARCH ARTICLE

\title{
REAL TIME VIDEO STREAMING USING HOLOGRAMS.
}

Prajakta Chavan, Gauri Bhatt, Neha Ture and Prof. K.K. Mathew.

Electronics and Telecommunication Department, Thadomal Shahani Engineering College.

\section{Manuscript Info}

\section{Manuscript History}

Received: 12 June 2017

Final Accepted: 14 July 2017

Published: August 2017

Key words:-

Holograms, Holographic video, Raspberry Pi, Voice over Internet

Protocol (VoIP), Pepper Ghost Effect,

Three Dimension, Video Conferencing.

\begin{abstract}
This paper implements a three dimensional method of live streaming which involves holograms. Holographic video streaming is the next generation of video streaming which just requires an additional holographic prism which acts as a display. To display a hologram on the prism, a holographic video is generated of the live stream using an HTML code. This project examines the new method of video transmission using a cost-effective system called raspberry pi and reception using an augmented reality technology of holographic projection. It presents the next level of video streaming using holograms as the medium, without using costly augmented reality gears. This improvement in the $2 \mathrm{D}$ technology can prove to be highly advantageous in the field of medical education. The system uses light diffraction from the screen which provides the holographic video to form a hologram on the prism. The result proves efficiency in transmission and reception of the live stream and also generates fine holograms.
\end{abstract}

Copy Right, IJAR, 2017,. All rights reserved.

\section{Introduction:-}

I.E. Sutherland quoted in his article "The Ultimate Display" in the year 1965 that "The ultimate display would, of course, be a room within which the computer can control the existence of matter. A chair displayed in such a room would be good enough to sit in. Handcuffs displayed in such a room would be confining, and a bullet displayed in such a room would be fatal. With appropriate programming, such a display could literally be the Wonderland into which Alice walked."

Holograms are 3D image projected in such a way that a viewer gets a feeling of seeing a live object. This is being widely used at presentations, seminars and places where 3D objects are presented to an audience. A hologram is not an image formed by a lens. Rather it is a photographic recording of a pattern of field of light which can be used to display entire three dimensional image of the subject under consideration, without any need of expensive eye gear or other special optics. The hologram itself is usually indiscernible when it is viewed under diffuse ambient light. It is an encoding of the light field as an interference pattern of seemingly randomness in the density, opacity or surface profile of the photographic medium.

Holographic video streaming provides ability to transmit information off the cell phone to create a hologram, projecting the video information into a real-life image. Real time holographic video streaming turns 2-D videos into

Corresponding Author:-K.K. Mathew.

Address:-Electronics and Telecommunication Department, Thadomal Shahani Engineering College. 
a real-world experience by just using a prism as a hologram projector. The video which is transmitted over IP is received by an IP device and projected as a hologram.

The transmission of the video is obtained using raspberry pi, a controller which is used to achieve efficient and effective audio and video transmission towards the receiver side. IP and pan-tilt-zoom cameras are used for deliverance of real time video remotely. Most of these IP cameras require high bandwidth thus involving this as a major disadvantage. Hence, it is necessary that low cost video acquisition units need to be developed. Such as a system that captures the real-time video images through a web camera and displays the capture video on the web browser with the help of IP. This real-time video can be seen in the browser of any display monitor or mobile devices with the help of a local host i.e. raspberry pi

\section{Functional Implementation}

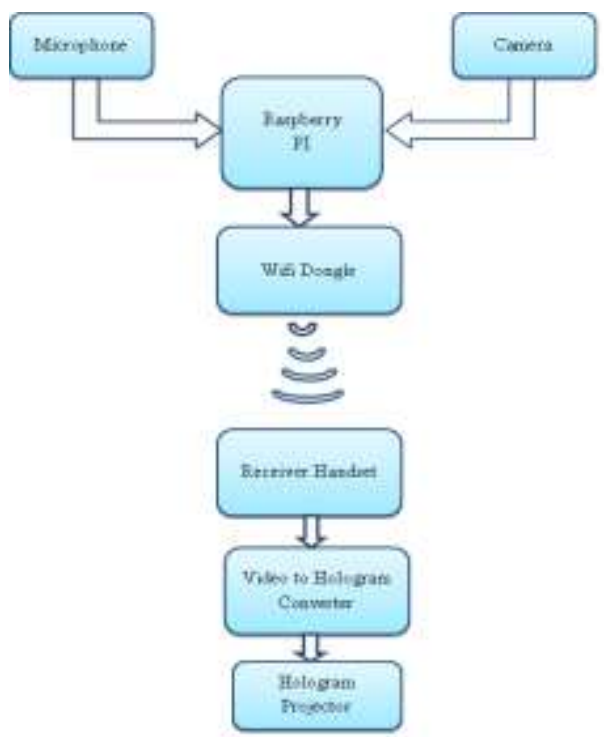

Fig. 1:- Block Diagram

The video to be captured by the webcam uses the software motion. It is used to enable the webcam to receive videos. Raspberry Pi is programmed using SSH (Secure Shell) via a laptop. Also the audio captured by the microphone of the webcam uses command "arecord" to record the audio. If a webcam with microphone is not used, then to work with another microphone a separate sound card is needed. A sound card is required to take the input from a USB microphone by the Pi. The sound card enables us to give an input to the Pi.

A Wi-Fi adapter is used to enable internet required for SSH and for transmitting and receiving videos over IP. Now the background of this audio-visual is removed using image processing. OpenCV software is installed in the PI to run the code for face recognition. This processed video is then transmitted using the Wi-Fi adapter as VoIP. The video will be transmitting in real time. This transmitted video is then received by any IP device. The video is received by entering the IP address and the web stream port number of the live stream. Then this received video will be converted to holographic video using the HTML language. The video is converted to the holographic video on the webpage itself where the URL of the receiving video is entered in the HTML code. Then placing a prism on the screen of a device where the holographic video is generated will project the hologram of the received video. These holograms will be viewed in real time from all four sides of the prism.

\section{Overview of the Raspberry Pi:-}

The processor at the heart of the Raspberry Pi system is a Broadcom BCM2835 system-on-chip (SoC) multimedia processor. This means that the vast majority of the system's components, including its central and graphics processing units along with the audio and communications hardware, are built onto that single component hidden beneath the $256 \mathrm{MB}$ memory chip at the centre of the board. It also uses a different instruction set architecture (ISA), known as ARM. The ARM-based BCM2835 is the secret of how the Raspberry Pi is able to operate on just 
the $5 \mathrm{~V} 1 \mathrm{~A}$ power supply provided by the onboard micro-USB port. It's also the reason why there aren't any heatsinks on the device as the chip's low power draw directly translates into very little waste heat, even during complicated processing tasks. [2]

Various generations of Raspberry Pi have been released. The first generation which was called Raspberry Pi 1 Model B was released in February 2012, before which a simpler and inexpensive model Model A was used. In 2014, a board with an improved design in Raspberry Pi 1 Model B+ was released by the foundation. These boards are approximately credit-card sized and represent the standard mainline form-factor. Improved A+ and B+ models were released a year later. A "compute module" was released in April 2014 for embedded applications, and a Raspberry Pi Zero with smaller size and reduced input/output (I/O) and general-purpose input/output (GPIO) capabilities was released in November 2015 for US \$5. The Raspberry Pi 2 which added more RAM was released in February 2015.

All models feature a Broadcom system on a chip (SoC), which includes an ARM compatible central processing unit (CPU) and an on-chip graphics processing unit (GPU, a VideoCore IV). CPU speed ranges from $700 \mathrm{MHz}$ to 1.2 GHz for the Pi 3 and on board memory range from $256 \mathrm{MB}$ to $1 \mathrm{~GB}$ RAM. Secure Digital (SD) cards are used to store the operating system and program memory in either the SDHC or MicroSDHC sizes. Most boards have between one and four USB slots, HDMI and composite video output, and a $3.5 \mathrm{~mm}$ phone jack for audio. Lower level output is provided by a number of GPIO pins which support common protocols like $\mathrm{I}^{2} \mathrm{C}$. The $\mathrm{B}$-models have an 8P8C Ethernet port and the Pi 3 and Pi Zero W have on board Wi-Fi 802.11n and Bluetooth.

\section{Holographic Display:-}

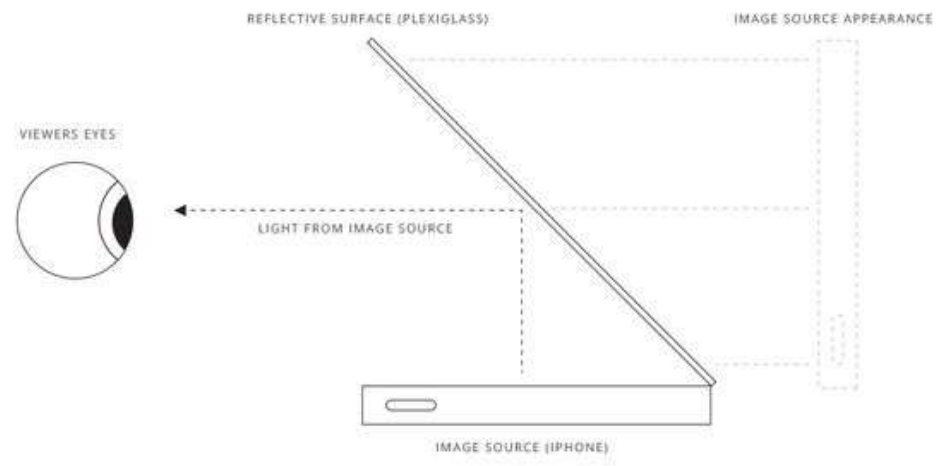

Fig. 2:- Pepper Ghost Effect

The hologram projector is based on the pepper ghost effect. Pepper's ghost is an illusion technique used in theater, amusement parks, museums, television, and concerts. The basic trick involves a stage that is specially arranged into two rooms, one that people can see into or the stage as a whole, and a second that is hidden to the side, the "blue room". A sheet of glass is placed somewhere in the main room at an angle that reflects the view of the blue room towards the audience. Generally this is arranged with the blue room to one side of the stage, and the plate on the stage rotated around its vertical axis at 45 degrees. Care must be taken to make the glass as invisible as possible, normally hiding the lower edge in patterning on the floor and ensuring lights do not reflect off it.

The reflected image cannot be seen when the lights are bright in the main room and dark in the blue room. When the lighting in the blue room is increased, often with the main room lights dimming to make the effect more pronounced, the reflection becomes visible and the objects within the blue room seem to appear in thin air. A common variation uses two blue rooms, one behind the glass and one to the side, which can be switched visible or invisible by alternating the lighting.

The hidden room may be an identical mirror-image of the main room, so that its reflected image matches the main rooms; this approach is useful in making objects seem to appear or disappear. This illusion can also be used to make one object or person reflected in the mirror appear to morph into another behind the glass (or vice versa). This is the principle behind the Girl-to-Gorilla trick found in old carnival sideshows. The hidden room may instead be painted 
black, with only light-colored objects in it. In this case when light is cast on the room, only the light objects reflect the light and appear as ghostly translucent images superimposed in the visible room. This can be used to make objects appear to float in space.

Advantages:-

- Video streaming using hologram provides a high resolution three-dimensional recording of an object.

- The use of existing augmented reality glasses or gears is very expensive for a small-scale application of holographic projection. Thus, it gives glasses free 3D display of objects.

- Holographic projection uses various techniques which projects the image into the surrounding space or environment. Thus, need for a projection screen, conventionally used for live video streaming is eliminated.

- A 3D view of an image having a considerable size provides a life like quality to the images, making them more real.

- The present research includes providing an interactive display to the holograms so that it could be accessible by the user.

- No need of any gears, absence of projection screen makes this system more economical and cost effective.

- Holographic images are perceived by the human eye with increased feasibility and depth of objects viewed, thus making them more lifelike.

- There is no requirement of special viewing devices unlike other 3D technologies

- Projecting an image using hologram provides a 360-degree view of the transmitted images or video, thus making the said technology more suitable for applications such as video conferencing.

\section{Applications and Future Scope:-}

A. Medical Applications

Holographic techniques are being used for three dimensional (3-D) rendering of medical pictures including MRI and CT pictures. Medical holographic imaging can enable doctors to test the insertion of medical instruments into an artificially constructed, three-dimensional version of the surgical field before the operation. Holographic projection can be used in medical education to project the organ under operation in real time 3-D manner in order to get the 360 degree view of the running operation. Thus, a surgeon can succeed in demonstrating a surgical procedure to medical students in person, without having to actually be there. [3]

\section{B. Holography in Education}

Education can be made more interesting and interactive in classrooms by making the teacher appear in classroom in the form of a hologram. This would make long distance learning by projection possible since the images are transmitted over the internet. Also, in demand education facilities could be made possible as it will allow the lecturer to be present in multiple lecture halls full of students at the same time. Highly sought-after experts could provide their expertise to a much wider audience, breaking down time and distance barriers.

\section{Marketing with 3D Holographic Display}

Holographic marketing is for advertising agencies and consumer products who wish to create a huge impact on the consumers through media. In January 2009 Coco-Cola gave a holographic sales conference presentation in Prague for over 800 people. Senior directors of the company were beamed into the stage as 3D holograms before giving a presentation about how the Coco-Cola brand has evolved over the years. The content of the presentation was also in the form of 3D holographic projections. The centre piece was a giant 3D hologram Coco-Cola branded spinning clock, representing the progression of time. A showcase of previous Coco-Cola bottles, logos, and labels amongst other objects were also projected as 3D holograms to create Prague's first 3D holographic projection display. [3]

\section{Holography in Entertainment Industry}

When one thinks about holography in the entertainment industry, the movies Star Trek and Star Wars come into mind. In these movies, people relate with holograms as they would relate with real human. Although, what people see in these movies are not real holograms, they depict what a real hologram looks like and future capabilities of holography. In the musical industry, holography is being used for concerts. In this case, the musicians can be far away in New York while performing in several cities around the world. Today, three-dimensional television and cinemas are becoming common, and there is more to come. 3D movies in home theatres require chunky glasses which may be uncomfortable for some people to wear. Also, experts found that viewing 3D television over a long 
period can cause headache and eye strain due to new sensory experience. Since holography makes beamed image look like real, it should not have any future strain on the eyes nor generate headache. [4]

\section{E. Holography in Science and Research}

With holographic technology, users can create holograms with almost any content, even outdoor scenes. Archaeologists, for example, already use optical holograms to archive and investigate ancient artifacts. Scientists can use hologram copies to perform their research without having access to the original artifacts or settling for inaccurate replicas. They can combine these holograms with interactive computer graphics to integrate real-time simulation data or perform experiments that require direct user interaction, such as packing reconstructed soft tissue into a fossilized dinosaur skull hologram. In addition, specialized interaction devices can simulate haptic feedback of holographic and graphical content while scientists are performing these interactive tasks. An entire collection of artifacts will fit into a single album of holographic recordings, while a light-box-like display such as that used for viewing X-rays can be used for visualization and interaction. This approach has the potential for wide applications in other industries. [5]

\section{Conclusions:-}

Using Holographic Technology for video viewing has endless applications, as far as human mind can imagine. In future, holographic displays will be replacing all present displays in all sizes, from small phones screen to large projectors. The performance of the system can be enhanced by considering certain conditions. This may include mounting of light sources to improve lighting. Holographic Technology and Spectral Imagining has endless applications, as far as the human mind can imagine. These technologies are indeed available and getting more robust in abilities each year. Not just through art or business communication, holographic technologies also represent education, safety, security, and most importantly planning and the strength of our civilization here and beyond. From entertainment to data visualization we can see a bright future for Holographic Projection and the bending and manipulation of light. Those areas of society which most often bring about research and development funding in technology are present amongst the many potential applications for this science. It therefore stands to reason and makes common sense that Holographic Technologies and Spectral Imaging will become a very integral part of human societies and civilizations in the future.

\section{References:-}

1. I. E. Sutherland, The ultimate display, Multimedia: From Wagner to virtual reality, 1965

2. Eben Upton and Gareth Halfacree, Raspberry Pi® User Guide, A John Wiley and Sons, Ltd.Publication, 2012

3. Sciences Ahmed Elmorshidy, "Holographic Projection Technology: The World is Changing.", PhD Journal of Telecommunications, Volume2, Issue2, May 2010

4. Aina, Oladapo. 2010, Application of Holographic Technology in Education, Bachelor's thesis, KemiTornio University of Applied Sciences

5. Bimber, Oliver, \& Raskar, Ramesh 2005, Spatial Augmented Reality Merging Real and Virtual Worlds. A K Peters, Wellesley, MA. 